\title{
Polarization control method for UV writing of advanced bragg gratings
}

Deyerl, Hans-Jürgen; Plougmann, Nikolai; Jensen, Jesper Bo Damm; Sørensen, Henrik Rokkjær; Kristensen, Martin

Published in:

Proceedings of 2002 IEEE/LEOS Workshop on Fibre and Optical Passive Components

Link to article, DOI:

10.1109/FOPC.2002.1015809

Publication date:

2002

Document Version

Publisher's PDF, also known as Version of record

Link back to DTU Orbit

Citation (APA):

Deyerl, H-J., Plougmann, N., Jensen, J. B. D., Sørensen, H. R., \& Kristensen, M. (2002). Polarization control method for UV writing of advanced bragg gratings. In Proceedings of 2002 IEEE/LEOS Workshop on Fibre and Optical Passive Components (pp. 86-91). IEEE. https://doi.org/10.1109/FOPC.2002.1015809

\section{General rights}

Copyright and moral rights for the publications made accessible in the public portal are retained by the authors and/or other copyright owners and it is a condition of accessing publications that users recognise and abide by the legal requirements associated with these rights.

- Users may download and print one copy of any publication from the public portal for the purpose of private study or research.

- You may not further distribute the material or use it for any profit-making activity or commercial gain

- You may freely distribute the URL identifying the publication in the public portal 


\title{
Polarization control method for UV writing of advanced
}

\section{Bragg gratings}

\author{
Hans-Jürgen Deyerl, Nikolai Plougman, Jesper Bo Jensen, Henrik Rokkjær \\ Sørensen, Martin Kristensen
}

COM, Technical University of Denmark, Building 345v, DK-2800 Kgs. Lyngby, Denmark e-mail: hjd@com.dtu.dk

\begin{abstract}
We report the application of the polarization control method for the UV writing of advanced fiber Bragg gratings (FBG). We demonstrate the strength of the new method for different apodization profiles, including the Sinc-profile and two designs for disperision-free square filters. The method has been tested for advanced FBGs with various strengths up to $65 \mathrm{~dB}$.
\end{abstract}

\section{INTRODUCTION}

As the demand for bandwidth in optical communication systems continues to grow much effort is put into a more efficient use of the available wavelength regions through the wavelength division multiplexing (WDM) technique. While the next generation electronic components will be able to handle $40 \mathrm{Gbit} / \mathrm{s}$ signals, the development in WDM systems is pushing the ITU channel spacing from $100 \mathrm{GHz}$ down to $50 \mathrm{GHz}$ and in future to $25 \mathrm{GHz}$. As the channel spacing decreases, the spectral properties of the individual components such as Bragg gratings becomes crucial. Bragg gratings working as channel selective components must have high bandwidth utilization and low out-of-band reflection in order to avoid cross-talk induced problems. In order to avoid an increasing bit-error rate when cascading several gratings the variation in the signal reflection and dispersion must also be small. The transmission dip and the side lobe suppression in the reflection spectrum must both exceed $30 \mathrm{~dB}$. Gratings with simple apodization profiles, such as Gaussian and Blackmann apodizations are not able to satisfy all these demands. The Sinc grating on the other hand has almost a square filter function with a flat stop band and little reflection outside this. Problems with dispersion can be reduced to acceptable levels by assymmetric modification of the Sinc profile [1]. Sinc gratings require several phase shifts, which is not possible to induce with the conventional dualscan method $\left[{ }^{2}\right]$. A frequently used method to write Bragg gratings with multiple phase shifts is the dithering method $\left[{ }^{3}\right]$ where fiber and/or phase mask are moved during the exposure. This method requires interferometric control of the relative position of the fiber and phase mask and is hence very sensitive to vibrations in the setup. We utilize a method, which is based on a spatial 
separation of the s- and p-polarizations of the UV beam [4]. Using this method we have been able to write up to $60 \mathrm{~dB}$ strong gratings with good agreement between simulations and written gratings.

\section{Principle}

The principle has been described previously. [4] Briefly a polarization beam splitter moves along the phase mask together with the UV beam from a $\mathrm{KrF}$ excimer laser $(248 \mathrm{~nm})$. Both fiber and phase mask are stationary during the exposure making the polarization control method less sensitive to vibrations than the dithering method. The polarization beam splitter introduces an angle between the propagation vectors of the s- and p-polarizations of the UV beam. In the fiber core the diverging beams write two spatially separated Bragg gratings. The phase shift between these gratings depends on the optical distance from the phase mask and the divergence angle $\theta$ of the polarization beam splitter. By adjusting the distance between phase mask and fiber, $d_{\text {air }}$, we can hence define arbitrarily phase shifts in the overall Bragg grating by shifting the UV intensity from one polarization to the other. A discrete phase shift of $\pi$ is obtained in the overall Bragg grating when $d_{a i r}$ is given by:

$4 \theta\left(d_{\text {air }}+r_{\text {fiber }} / n_{\text {clad }}\right)=\Lambda_{\text {mask }}$

where $r_{\text {fiber }}$ is the fiber radius, $n_{\text {clad }}$ is the refractive index of the fiber cladding at $248 \mathrm{~nm}$ and $\Lambda_{\text {mask }}$ is the phase mask period. The divergence angle is assumed to be small, so that the assumption $\sin \theta \sim \tan \theta \sim \theta$ is justified. The beam splitter used in our setup is a Wollaston prism with a divergence angle of 0.14 degrees. With a fiber diameter of $125 \mathrm{~mm}, d_{a i r}$ is in the order of $68 \mu \mathrm{m}$ for a Bragg grating with a center wavelength around $1550 \mathrm{~nm}$. Adjustment of this distance must be very accurate in order to obtain high quality gratings. The ratio of UV intensity in the two polarizations is controlled by a polarizer mounted on a computer controlled rotation stage. Denoting the angle of the polarizer relative to the spolarization, the fluences in the two polarizations are $F_{s}=F \cos ^{2} \alpha$ and $F_{p}=F \sin ^{2} \alpha$ respectively, where $F$ is the total fluence of the beam. Due to the discrete $\pi$ phase shift between the two polarizations the UV induced refractive index modulation strength $\Delta \mathrm{n}(\mathrm{z})$ in the resulting Bragg grating is described by

$\Delta n(z)=\eta F+\eta F \cos (2 \alpha) \cos \left(\frac{2 \pi z}{\Lambda_{\mathrm{B}}}+\phi_{0}\right)$

where $\mathrm{z}$ is the position along the fiber, $\phi_{0}$ is a constant and $\eta$ describes the photosensitivity of the fiber. Equation 2 shows that the change in the effective refractive index, $\eta \mathrm{F}$, is constant along the fiber, while the modulation amplitude scales with $\cos (2 \alpha)$. Thus by rotating the polarizer we are able to change the 
modulation strength while maintaining a constant effective refractive index. Maximum modulation strength is obtained when the polarizer angle is either $0^{\circ}$ or $90^{\circ}$, that is when only one polarization is present, while zero modulation is achieved when $\alpha=45^{\circ}$ and the UV intensity in the s-polarization equals that in the p-polarization. The wavelength of the resulting Bragg grating is $\lambda_{B}=n_{\text {eff }} \Lambda_{\text {mask }}$, where $n_{\text {eff }}$ is the effective refractive index after the exposure.

\section{EXPERIMENTAL RESULTS AND DISCUSSION}

Applying Fourier theory for the design of fiber Bragg gratings is only justified for weak gratings. When dealing with the response of strong FBGs, e.g. when the light in the stop band no longer penetrates the full length of the modulation profile without any significant attenuation, Fourier theory cannot be applied for the full analysis of the spectral response of the FBG. Deviations from the Fourier analysis appear when higher strength FBG with certain properties, e.g. low dispersion, are designed. Several approaches, which are based on inverse scattering techniques have been developed to address this issue. $[5,6]$

\subsection{Sinc grating}

The inverse Fourier transform of a square filter function is a Sinc index modulation profile. The normalized apodization profile for writing a Sinc grating is seen in Figure 1(a), while Figure 1(b) shows the corresponding polarizer angle profile. The Sinc grating is convoluted with an additional Gaussian apodization to avoid problems due to the finite length of the grating. This reduces the steepness of the sides of the reflection peak, but is necessary to obtain strong side lobe suppression as may easily be seen from simulations. As indicated in Figure 1 we obtain a $\pi$ phase shift in the grating whenever the polarizer angle crosses over the $\alpha=45^{\circ}$ line, which is marked with a dotted line in the figure 1. 

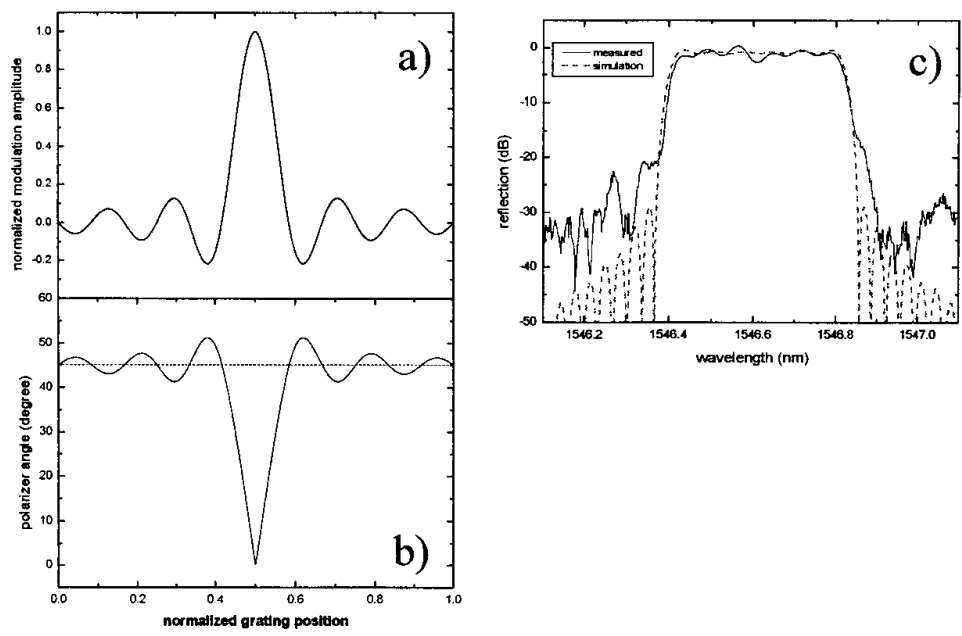

Figure 1. (a) Normalized modulation amplitude and (b) corresponding polarizer angle for a Sinc grating. The dashed line at $\alpha=45^{\circ}$ marks the cross over between segments of the grating with different phase. Reflection spectrum (c) for a $23 \mathrm{~mm}$ long weak Sinc-grating written in deuterium loaded TW-fiber.

Figure 1c shows the measured and simulated (dashed line) reflection spectrum of a $23 \mathrm{~mm}$, long weak (transmission loss $<0.15 \mathrm{~dB}$ ) Sinc grating written in a deuterium loaded TW-fiber. The reflection spectrum shows a side lobe suppression of $25 \mathrm{~dB}$ and is in very good agreement with the Fourier analysis.

\subsection{Tailored gratings}

The target design of the linear phase (dispersion free) Bragg grating shown here includes zero dispersion throughout the spectral response. The grating was designed to have a constant transmission-loss of $20 \mathrm{~dB}$ over the full drop window. The modulation profile was generated by solving the inverse scattering problem by the layer peeling algorithm implemented in the commercial program IFO_Gratings [7]. The theoretical normalized modulation amplitude is shown in figure 2(a) along with the corresponding polarizer angle $\alpha$ in figure 2(b). 

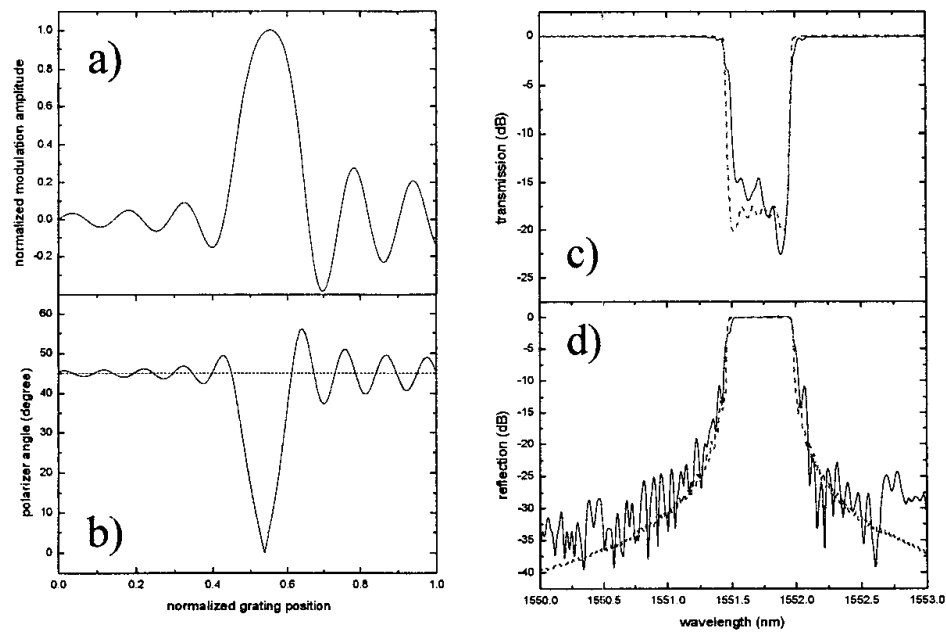

Figure 2. (a) Normalized modulation amplitude and (b) corresponding polarizer angle for a $99 \%$ reflectivity grating. The dashed line at $\alpha=45^{\circ}$ marks the cross over between segments of the grating with different phase. Transmission (a) and reflection (b) spectra for a $23 \mathrm{~mm}$ long FBG written in a deuterium loaded HNLF fiber.

Fig. 2 shows the measured transmission (c) and reflection (d) spectra. The simulations based on coupled mode theory are in good agreement with the experimental results. The spectra show also good side lobe suppression down to $15 \mathrm{~dB}$ at a transmission dip of $20 \mathrm{~dB}$.

A second low-dispersion design with a higher transmission loss $(65 \mathrm{~dB})$ has been written. The results are shown in figure 3.
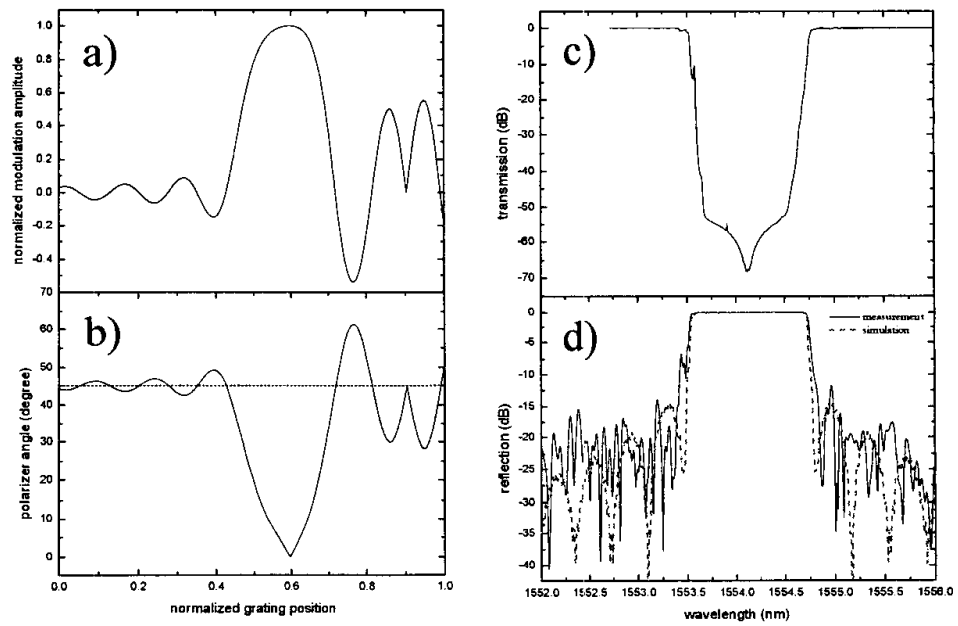

90 
Figure 3. (a) Normalized modulation amplitude and (b) corresponding polarizer angle. The dashed line at $\alpha=45^{\circ}$ marks the cross over between segments of the grating with different phase. Transmission (a) and reflection (b) spectra for a $23 \mathrm{~mm}$ long FBG written in a deuterium loaded HNLF fiber.

The measured specrtra are in very good agreement with the simulated spectra and show a side lobe suppression of $15 \mathrm{~dB}$ at a maximum transmission dip of $65 \mathrm{~dB}$.

\section{CONCLUSION}

High reflection near square pass band linear fiber Bragg gratings have been written with the novel polarization controlled method. The presented results show the strength of the new writing method for the inscription of Bragg gratings including phase shifts from weak to very strong FBGs. The gratings were designed solving the inverse scattering problem for the tailoring of nonuniform Bragg gratings. Dispersion measurements on these special designs are currently under way.

\section{REFERENCES}

$\left.{ }^{1}\right] \quad$ M. Ibsen, P. Petropoulos, M. N. Zervas, R. Feced, "Dispersion-free fibre Bragg gratings", in OSA Trends in Optics and Photonics (TOPS) vol. 54, MC1-12001.

$\left.{ }^{2}\right] \quad$ R. Kashyap, A. Swanton, D. J. Armes, "Simple method for apodising chirped and unchirped fibre Bragg gratings", Electron. Lett. vol. 32, no. 13, pp. 1226-1228, 1996.

[3] M. J. Cole, W. H. Loh, R. I. Laming, M. N. Zervas, and S. Barcelos, "Moving fibre/phase mask-scanning beam technique for enhanced flexibility in producing fibre gratings with uniform phase mask", Electron. Lett. vol. 31, no. 17, pp. 1488-1490, 1995.

$\left[{ }^{4}\right] \quad$ J. B. Jensen, N. Plougmann, H.-J. Deyerl, P. Varming, J. Hübner and M. Kristensen, "Polarization control method for UV writing of advanced Bragg gratings", Opt. Lett., in press.

[ $\left.{ }^{5}\right] \quad$ R. Feced, M. N. Zervas, M. A. Muriel, "An efficient inverse scattering algorithm for the design of nonuniform fiber Bragg gratings", IEEE J. Quantum Elect. vol. 35, no. 8, pp. 1105-1115, 1999.

[6] L. Poladian, "Simple grating sythesis algorithm", Opt. Lett., vol. 25, no. 11, pp. 787-789, 2000.

[7] IFO_Gratings, Version 4.0 by Optiwave Corporation (http://www.optiwave.com) 